\title{
Feature-enhanced Surfaces from Incomplete Point Cloud with Segmentation and Curve Skeleton Information
}

\author{
Meili Wang, Yuling Fan \\ College of Information Engineering \\ Northwest A \& F University \\ Yangling, China \\ Email:wml@nwsuaf.edu.cn \\ Shihui Guo $\bowtie$, Minghong Liao \\ School of Software \\ XiaMen University \\ XiaMen, China \\ Email: guoshihui@xmu.edu.cn \\ Dongjian He \\ College of Mechanical and Electronic Engineering \\ Northwest $A \&$ \& $F$ University \\ Yangling, China
}

\author{
Jian Chang \\ National Centre for Computer Animation \\ Bournemouth University \\ Bournemouth, UK
}

\begin{abstract}
Raw data of point cloud is often noisy and with topological defects (such as holes), which cause problem$s$ including faulty connection and inaccurate structure. As a result, the surface reconstruction of point cloud data is a highly challenging problem. This work proposes a novel method, which improves the surface quality compared with existing methods. Our method combines both the local detailed features and the global topological information during the reconstruction process. To facilitate the feature refinement, we first pre-process the point cloud data by relocating each point, upsampling the point data, and optimizing normals to enhance the features and geometric details. We then identify the topological information by segmenting the geometry and constructing curve skeletons for each part and guide the surface reconstruction with the skeletons by minimal user interaction. We demonstrate the effectiveness of our methods with various examples, where our reconstruction can fill out missing data and preserve sharp features.
\end{abstract}

Keywords-Surface reconstruction; Segmentation; Enhanced features; Curve skeleton

\section{INTRODUCTION}

Fully developed three-dimensional (3D) scanners can now produce $3 \mathrm{D}$ point cloud representing increasingly complex models, which is an important data source of computer graphics research. Nowadays, point cloud is easily acquired for our purposes, but the data are usually noisy, sparse, and unorganized. A large amount of research effort and attention have been paid to the reconstruction of meaningful surface models from point cloud in the past three decades $[1,2]$. However, surface reconstruction from incomplete point cloud is still a challenging and unresolved problem.

Our work is inspired by the observation that the points of one division share the same characteristics and represent a substructure. Segmentation is considered useful for analyzing various aspects of a scene such as locating and recognizing parts, classification, and feature extraction. Although there are many segmentation [3] and reconstruc- tion algorithms, there is no reconstruction methods use the segmentation information in surface reconstruction and few methods are for incomplete point cloud. Meanwhile, the goal of user-interactive editing in the pipeline of surface reconstruction is also compromised in the majority of existing works.

Based on the aforementioned motivations, we propose a novel method that integrates both segmentation and curve skeletonization to accomplish the goal of surface reconstruction from incomplete point cloud. First, the point cloud data undergoes the procedure of data pre-processing. After preprocessing, we conduct a segmentation operation considering spatial and geometric features of a model to create meaningful point cloud divisions or subsets. Last, curve skeletons are extracted for individual segments and used as new editing handles and features for improving fine detail reconstruction.

The aim of our work is to extend the previous success of point cloud segmentation into our surface reconstruction, which has been used for hole filling and template-based surface reconstruction.

\section{RELATED WORK}

\section{A. Point cloud segmentation}

3D point cloud segmentation classifies parts of point cloud with the same properties into the same region. [2] used a 3D moving fovea to process parts of a scene with different levels of resolution, which helped recognize and identify objects in point cloud. [4] defined the reconstruction task as a labeling problem. They applied point cloud segmentation to define content into rooms and the outside area as a pre-processing filter to accelerate their computing and simplify the processing, which inspired our approach of applying segmentation for full reconstruction. To show the effectiveness of $3 \mathrm{D}$ point cloud segmentation, we utilized the segmentation results as a pre-processing step for final reconstruction. 


\section{B. Curve skeletons}

A few methods [5, 6] have been developed for curve skeleton extraction from incomplete point cloud. [5] evolved deformable snakes based on surface tension control or smoothness priors for topological and geometric reconstruction. [7] extended curve skeletons with medial sheets to reconstruct noncylindrical geometry. In existing works, surface reconstruction with incomplete data does not primarily consider sharp features. Inspired by the fact that a curve skeleton is an abstraction of data that can tolerate missing data, we have extended the existing method to radical cases in which a large amount of data is missing. Using the segmentation results, our approach takes advantages of allowing simple user interaction for guidance cues preserve and enhance sharp features.

\section{C. $3 D$ reconstruction}

The task of reconstruction is from point cloud to automatically construct mesh models, which are convenient to store, edit, render, and animate. The core aims of reconstruction are to recover missing data and preserve model shapes. In the past 20 years, surface reconstruction technology has made considerable progress [1], ranging from explicit reconstruction to implicit function-based reconstruction. Most recent methods have focused on priori knowledge oriented specific model reconstruction and interactive reconstruction in response to serious deficiencies in point cloud. [4] presented an automatic approach to the parametric reconstruction of 3D building models from indoor point cloud. It took human inputs as a first guess, and later treated the reconstruction problem as a labeling problem that was solved by energy minimization. [8] introduced a statistical model using a Poisson distribution to extract feature points from point cloud. In contrast to the model in [4], their approach did not require any prior knowledge of the surface. Their reconstruction is affected by noise and sampling quality, which we address in our approach to enhancing the overall performance of reconstruction.

\section{PRE-PROCESSING}

\section{A. Simplification}

A new point cloud set which reflects information of underlying shape by improving local projection operator(LOP) [9] is conducted.

Given the original captured point set $P=\left\{p_{j}\right\}_{j \in J} \subset$ $R^{3}$, the LOP algorithm projects an arbitrary point set $X=$ $\left\{x_{i}\right\}_{i \in I} \subset R^{3}$ onto the original captured set $P$, and then minimizes the sum of distances from projected set $Q=$ $\left\{q_{i}\right\}_{i \in I}$ to set $P$.

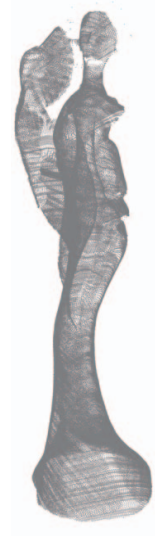

(a)

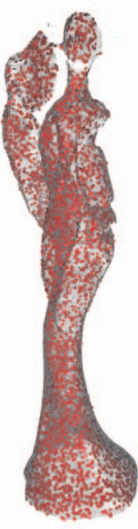

(b)

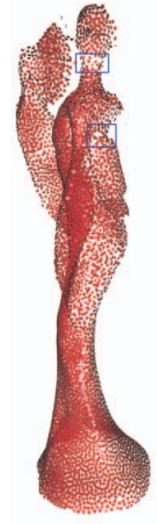

(c)
Figure 1. The disadvantage of LOP on the Japanese lady. (a) Raw data. (b) Arbitrary points in the raw data set. (c) LOP.

We improve LOP in two ways to overcome its disadvantages: (i) If the raw data is highly non-uniform, the projected results will also be nonuniform, an illustration of Figure 1 is used to show the disadvantage. In Figure 1, it is obvious that the result is nonuniform in the rectangle. To address this problem, we use adaptive density weights in LOP. (ii) The operation is still isotropic because it does not consider sharp features. To preserve sharp features, we import anisotropic neighbors into the first term of the LOP. Finally, our improved LOP algorithm updates each $q_{i}^{(k)} \in Q^{(k)}$ in iteration $k$ using the following equations. The first iteration is:

$$
q_{i}^{(1)}=\frac{\sum_{j \in J} p_{j} \varsigma\left(\mathbf{n}_{j}, p_{j}-q_{i}^{(0)}\right)}{\sum_{j \in J} \varsigma\left(\mathbf{n}_{j}, p_{j}-q_{i}^{(0)}\right)}
$$

We define the weighted densities for each point $p_{j}$ in $P$ and $q_{i}$ in $Q$ during the iteration $k$ by

$$
\begin{aligned}
& \rho_{j}=1+\sum_{j^{\prime} \in J \backslash\{j\}} \varsigma\left(\mathbf{n}_{j}, p_{j}-p_{j^{\prime}}\right) \\
& w_{i}^{(k)}=1+\sum_{i^{\prime} \in I \backslash\{i\}} \theta\left(\left\|q_{i}^{(k)}-q_{i^{\prime}}^{(k)}\right\|\right), k=0,1,2, \ldots \ldots .
\end{aligned}
$$

where $\mathbf{n}_{j}$ is the normal vector of the point $p_{j}$. The normal vector can be calculated by PCA. $\rho$ and $w$ are the local adaptive weighted densities, they can allow higher densities near sharp features. Finally, the projected result for $q_{i}^{(k)}$ can then be defined as

$$
\begin{aligned}
& q_{i}^{(k+1)}=\sum_{j \in J} p_{j} \frac{\alpha_{j}^{i} / \rho_{j}}{\sum_{j \in J} \alpha_{j}^{i} / \rho_{j}}+\mu \sum_{i^{\prime} \in I \backslash\{i\}}\left\|q_{i}^{(k)}-p_{j}\right\| \frac{\sum_{i^{\prime} \in I \backslash\{i\}} w_{i^{\prime}}^{(k)} \beta_{i^{\prime}}^{i}}{w_{i^{\prime}}^{(k)} \beta_{i^{\prime}}^{i}} \\
& \alpha_{j}^{i}=\frac{\theta\left(\left\|q_{i}^{(k)}-p_{j}\right\|\right)}{\left\|q_{i}^{(k)}-p_{j}\right\|} \\
& \beta_{i^{\prime}}^{i}=\frac{\theta\left(\left\|q_{i}^{(k)}-q_{i^{\prime}}^{(k)}\right\|\right) \eta^{\prime}\left(\left\|q_{i}^{(k)}-q_{i^{\prime}}^{(k)}\right\|\right)}{\left\|q_{i}^{(k)}-q_{i^{\prime}}^{(k)}\right\|}
\end{aligned}
$$


where $\|$.$\| is the L_{2}$-norm, and $\mu$ is the weight parameter which used to balance attraction and repulsion. Further, $\rho$ and $w$ are the adaptive density weights, and $\varsigma$ is used to consider the normal directions that can be used to indicate approximately where the edges indicate sharp features. In our experiments, we set $\mu=0.4, \eta(r)=-r$. Further, $\theta$ and $\varsigma$ can be expressed as follows:

$$
\begin{aligned}
& \theta(r)=e^{-r^{2} /(h / 4)^{2}} \\
& \varsigma\left(\mathbf{n}_{j}, p_{j}-p_{j^{\prime}}\right)=e^{-\left(\mathbf{n}_{i}^{\mathrm{T}}\left(p_{j}-p_{j^{\prime}}\right)\right)^{2} / \sigma_{p}^{2}} \\
& \sigma_{p}=\operatorname{dist}_{b b} / \sqrt{|J|}
\end{aligned}
$$

where parameter $\theta$ is a rapidly decreasing smooth weight function with support radius $h$, that defines the size of the influence neighborhood and can be adjusted adaptively. We use a default value of $h=4 \sqrt{d i s t_{b b} /|J|}$ where dist is the diagonal length of set $P$ 's bounding box.

Figure 2 shows the results of simplifying a point cloud using the proposed method compared to the results obtained by [9]. In our method, owing to the density weights $\rho$ and $w$, the attraction is relaxed by $\rho$ with respect to the attractive energy, and the repulsion in dense areas is strengthened by weight $w$ with respect to the repulsive energy. Hence, our algorithm obtains more uniformly spaced points than LOP.

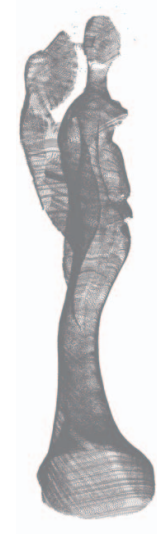

(a) Raw data

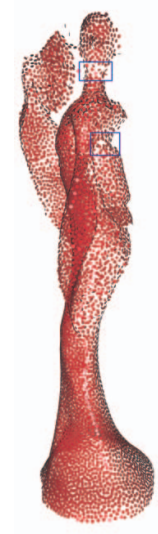

(b) LOP [9]

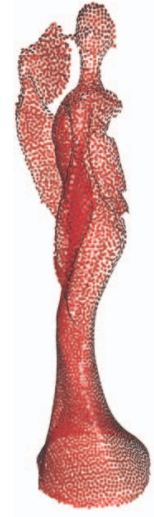

(c) Ours
Figure 2. Point cloud simplified by 20 iterations

\section{B. Feature enhancement}

In this paper, an improved edge-preserving upsampling (EAR) [10] is used to enhance features. For each insertion, this method adds a new point $b_{k}+d_{k} \mathbf{n}_{k}$ in two steps: finding the near-sparsest insertion base $b_{k}$, and optimizing the projection distance $d_{k}$ to move the point onto the latent surface.

Considering an existing point $s_{i}$ and its neighbors in the

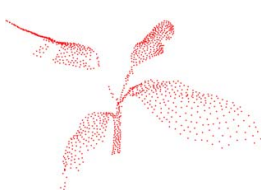

(a) Simplified points

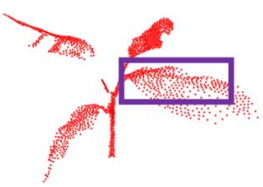

(b) EAR in [10]

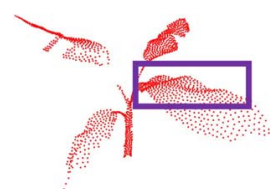

(c) Our method
Figure 3. Upsampling results of EAR and our method.

set $N_{s i}$, the EAR inserts a new point using:

$$
\begin{aligned}
& \text { maximizes } C(b)=\min _{s_{i^{\prime}} \in N_{s i}}\left\|b-s_{i^{\prime}}-\mathbf{n}_{i^{\prime}}^{T}\left(b-s_{i^{\prime}}\right) \mathbf{n}_{i^{\prime}}\right\| \\
& \sum_{s_{i} \in N_{b_{k}}}\left(\mathbf{n}^{T}\left(s-s_{i}\right)\right)^{2} \theta\left(\left\|s-s_{i}\right\|\right) \varphi\left(\mathbf{n}-\mathbf{n}_{i}\right)
\end{aligned}
$$$$
\text { where } \varphi\left(\mathbf{n}, \mathbf{n}_{i}\right)=e^{-\left(\frac{1-\mathbf{n}_{i}^{\mathrm{T}} \mathbf{n}_{i^{\prime}}}{1-\cos \alpha}\right)^{2}}, b \text { is the base location, } s_{i}
$$
is an existing point, set $N_{s_{i}}$ is its neighbors, $\theta$ can be got from Equation (4).

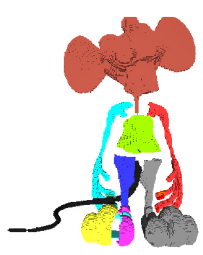

(a)

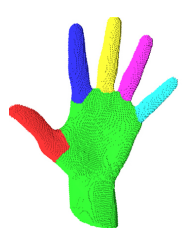

(b)

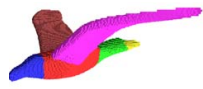

(c)

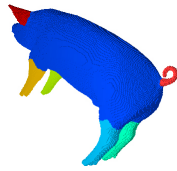

(d)
Figure 4. Segmentation results

Moreover, to minimize the weighted total projection distance and add new points along the sharp edges, we introduce the anisotropic neighbors to enhance features. Finally, the calculation of projection distance using our method is as follows:

$$
d_{k}\left(b_{k}, \mathbf{n}\right)=\frac{\sum_{s_{i} \in N_{b_{k}}}\left(\mathbf{n}^{\mathrm{T}}\left(b_{k}-s_{i}\right)\right) \varsigma\left(\mathbf{n}, b_{k}-s_{i}\right) \varphi\left(\mathbf{n}, \mathbf{n}_{i}\right)}{\sum_{s_{i} \in N_{b_{k}}} \varsigma\left(\mathbf{n}, b_{k}-s_{i}\right) \varphi\left(\mathbf{n}, \mathbf{n}_{i}\right)}
$$

where $\varphi$ can be got from Equation (5).

Figure 3 compares the results of EAR in [10] and our method. As we can see, EAR inserts some points evenly along some edges, while our method inserts points that maintain sharper edges.

\section{SURFACE RECONSTRUCTION}

\section{A. Segmentation}

Point cloud segmentation is one of the key technologies for 3D reconstruction. In this paper, we use a self-adaptive segmentation algorithm, this algorithm consists of two main steps: automatic selection of the centers according to the extracted features and segmentation of the points according to the centers [11].

In the process of center selection, a center has a high density compared to its surrounding neighbors with lower 


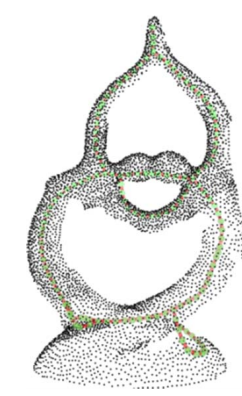

(a)

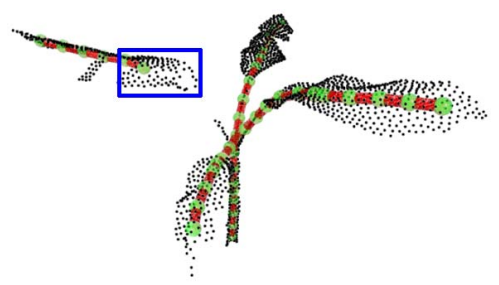

(e)

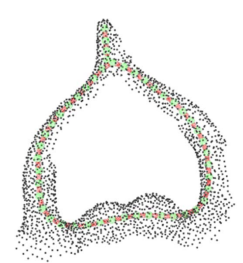

(b)

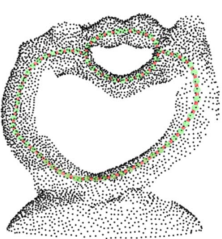

(c)

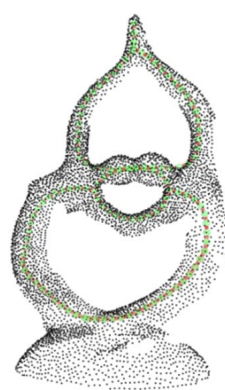

(d)

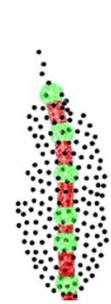

(f)

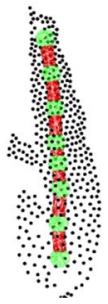

(g)

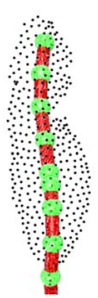

(h)

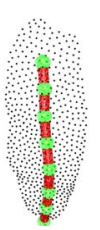

(i)

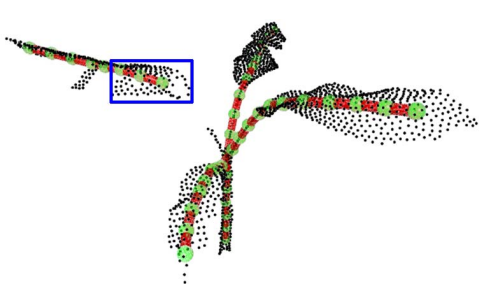

(j)

Figure 5. Curve skeletons: (a) and (e) skeleton of unsegmented points, (b)-(c) and (f)-(i) parts of the skeleton, and (d) and (j) skeleton of segmented point cloud.

density and a large diversity with respect to other centers. The centers can be measured by representativeness value and diversity value.

Representativeness can be measured by a point that has a higher density than its neighbors. The diversity can be measured by computing the minimum distance between a point and other points with densities that are higher than that of a point. We determine the centers using Equation (10):

$$
\begin{aligned}
& s p_{i}^{A t t}=\log \nu_{i}+\log \delta_{i} \\
& \nu_{i}^{\text {Rep }}=1+\sum_{i^{\prime} \in I} \theta\left(\left\|p_{i^{\prime}}-p_{i}\right\|\right) \\
& \delta_{i}^{D i v}=\min _{i^{\prime}<i}\left\|p_{i^{\prime}}-p_{i}\right\|
\end{aligned}
$$

where $\delta$ is the diversity value, $\nu$ is the representativeness value, and $\theta$ is the same as defined in Equation (4).

After obtaining the centers, each remaining point is assigned to the same cluster as its nearest neighbor of higher density. Using this method, the segmentation can be completed, and the results of segmentation are shown in Figure 4.

\section{COMPARISON AND ANALYSIS}

\section{A. L1-curve skeleton}

To obtain the geometric representation, the L1-medial skeleton [6] is used to represent the curve skeleton. It iteratively projects points to produce the L1-medians of the local neighborhoods. We use the following definition to obtain a set of projected points $M=\left\{m_{l}\right\}_{l \in L}$ in each segmented clusters:

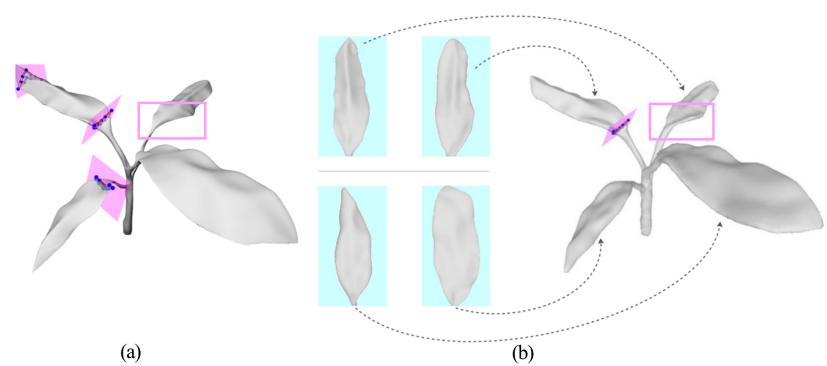

Figure 6. Reconstruction of the plant model using our method compared with the Morfit results. (a)Morfit results, (b) results of our method and each part of the segmented reconstruction.

$$
m_{l}^{k+1}=\frac{\sum_{i \in I} z_{i} \alpha_{i}^{l}}{\sum_{i \in I} \alpha_{i}^{l}}+\mu \sigma_{l}^{k} \frac{\sum_{l^{\prime} \in L \backslash\{l\}}\left(m_{l}^{k}-m_{l^{\prime}}^{k}\right) \beta_{l^{\prime}}^{l}}{\sum_{l^{\prime} \in L \backslash\{l\}} \beta_{l^{\prime}}^{l}}
$$

where

$$
\beta_{l^{\prime}}^{l}=\frac{\theta\left(\left\|m_{l}^{k}-m_{l^{\prime}}^{k}\right\|\right)}{\left\|m_{l}^{k}-m_{l^{\prime}}^{k}\right\|^{2}}, \alpha_{i}^{l}=\frac{\theta\left(\left\|m_{l}^{k}-m_{i}^{k}\right\|\right)}{\left\|m_{l}^{k}-m_{i}^{k}\right\|},
$$

$\sigma_{l}=\frac{\lambda_{l}^{2}}{\lambda_{l}^{0}+\lambda_{l}^{1}+\lambda_{l}^{2}}, \lambda$ is eigenvalue, and $\lambda_{l}^{0} \leq \lambda_{l}^{1} \leq \lambda_{l}^{2}$.

The aforementioned iterative projection produces a set of points $M=\left\{m_{l}\right\}_{l \in L}$, which can form a skeleton of the underlying shape. The final results of the L1-curve skeleton are shown in Figure 5. As shown in rectangle, our skeleton is similar to the original shape. We compare the unsegmented with segmented results to demonstrate our results, where the topology of the models has been improved. 


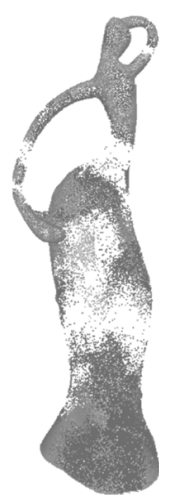

(a) Raw data

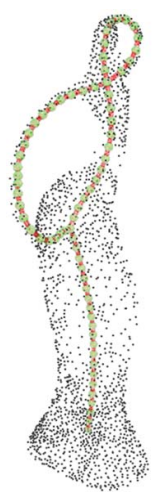

(b) Skeleton

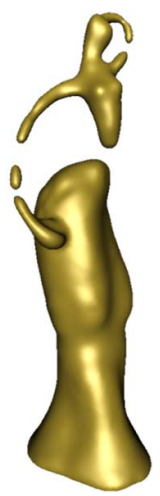

(c) Poisson

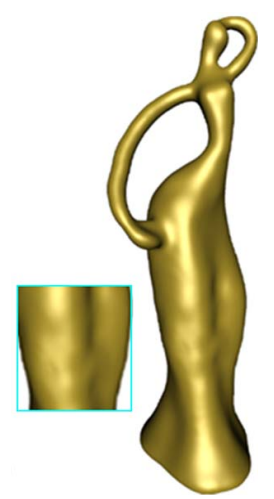

(d) Morfit

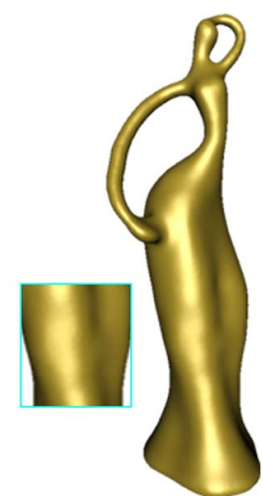

(e) Ours

Figure 7. Results comparison on lady model

\begin{tabular}{|c|c|c|c|c|}
\hline \#Fig & Lady(Morfit) & Lady(Our) & Plant(Morfit) & Plant(Our) \\
\hline \#BE & 0 & 0 & 0 & 0 \\
\hline \#CE & 0 & 0 & 3 & 0 \\
\hline \#PS & 3 & 1 & 4 & 1 \\
\hline \#SS & 0 & 0 & 0 & 0 \\
\hline
\end{tabular}

Table I

COMPARISON OF THE NUMBER OF USER INTERACTION [12]. \#BE: NUMBER OF SKELETAL BRANCHES EDITED/ADDED/REMOVED; \#CE: NUMBER OF PROFILE CURVES EDITED; \#PS: NUMBER OF STROKES TO INDICATE SWEEP PATHS; \#SS: NUMBER OF SHARPENING STROKES.

After getting the skeleton of data, we applied an interactive Morfit [12] to reconstruct each part of the segmentation. The separate parts are fused as an ensemble of general cylinders around the curve skeleton. The Morfit algorithm allows users to edit the model on a larger scale. For the L1-center skeleton of the model, users can adjust the model by disconnection, connection, pruning, extension, and deformation, and hence unambiguously express the model topology. We performed them for each part of the model and obtained the reconstructed results of each part using Morfit.

Figure 6 contrasts the effects of using our method and Morfit of a plant model obtained from [6], and Table 1 gives the number of interactions for this model. The improvement of the models is clear. For the plant model, Figure 6(b) shows the reconstructed results of each part after the segmentation of the point cloud. The algorithm keeps the sharp features well which are highlighted in the pink rectangle compared with the highlighted in the same part of the Morfit algorithm in Figure 6(a). In Figure 6(a), it can be seen that the blade are smooth and similar, the reconstruction results of them have minor difference. In addition, the number of human interaction of our proposed method is less than that of Morfit, and the efficiency of reconstruction is also improved.

\section{B. Comparison}

In this section, we show the reconstruction results obtained by our method on models compared with those obtained by other methods.

In Figure 7, the results of the lady model show that our algorithm can deal with incomplete point cloud (Figure 7(e)), which provides the most faithful reconstruction result with only one path stroke and editing. Figure 7 (b) is the extracted skeleton model, which better represents the model. Figure 7(c) is the model reconstructed using the Poissonbased algorithm directly from the original point cloud. This is less adaptable to the incompleteness of the point cloud. Figure $7(\mathrm{e})$ is the result of reconstruction according to the result of Figure 7(b). From Figure 7(d) and Figure 7(e), for the left arm of the lady body, our reconstruction results are more slim, the shape is better fitting to the original complete model. And for the enlarged part, our reconstruction results are smoother.

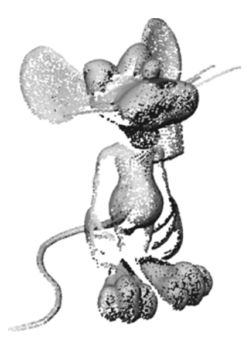

(a) Raw data

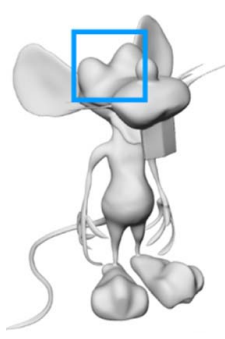

(b) Morfit

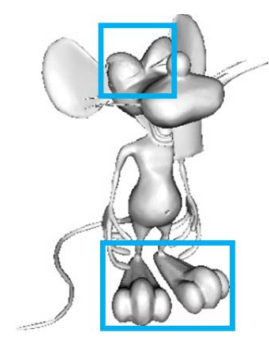

(c) Ours
Figure 8. Reconstructed model according to different parts adopting different algorithms

In Figure 8, we reconstructed the mouse model to demonstrate the effects of segmentation and feature enhancement. We implemented and compared Morfit's method and ours. The method of Morfit is to reconstruct the model directly, and ours is divided into two processes. The first process is to divide the model. The second process reconstructs the segmented model for different segmentation part. In the model, after segmentation, the parts of the eye and toe were applied with Poisson reconstruction, and others were applied with Morfit. As can be seen from Figure 8(b), the 
reconstruction of the eyes part can show the details of the orbit, the reconstruction results of toe also have clear edges and distinct contours. Obviously, the details of the result are closer to the original model and represent the surface change more real.

\section{CONCLUSION}

This work constructs a mesh surface from a point cloud, with its advantages in preserving fine details, handling incomplete data and allowing flexible user interactions compared with existing works. Our method first includes a preprocessing step, which improves the quality of point cloud distribution and enhances sharp features, and next conducts the curve-skeleton-based reconstruction, which smooths the surface according to the extracted 1D structure. In the pre-processing, our resampling simplification improves the quality of the point distribution with uniform density using attractive and repulsive forces. It also improves the LOP with a customized anisotropic operator and is capable of processing highly nonuniform distributions to produce satisfactory results in cases where LOP may fail.

The feature enhancement is achieved via two approaches. One is EAR to add new points near the sharp features to better preserve their shapes. The other optimizes the normal distribution considering the anisotropic features with different point clusters, distinguishing normals across discontinuities.

Segmentation and 1D curve skeletons are adopted for surface extraction. Thus, building a complex shape can be divided into many simple subsets of building the parts with regular/simple shapes. Our approach requires fewer user interactions without degenerating the quality and efficiency of the output. For the final outputs, our results present more feature details, whereas existing methods such as the Poisson-based method and Morfit tend to overfit the surface and smooth out small details. The limitations of the proposed method are its dependence on the extraction of representative curve skeletons. If the models have a highly complex structure/shape, the method requires several user edits and obtains many segments. Our future work is to investigate the case of objects with complex shapes and identify suitable solutions that are effective in terms of both usability and accuracy.

\section{ACKNOWLEDGMENT}

This work was partially funded by the National Natural Science Foundation of China (61402374, 61702433), the National High-tech research and Development Program (863 Program: 2013AA10230402), China Postdoctoral Science Foundation (2014M562457), China Postdoctoral Science Foundation (2016M600506) and 1st NRF-NSFC Joint Research Grant (6161101193). The authors acknowledge Shenzhen Key Lab of Visual Computing and Visual Analytics for the source data and the models.

\section{REFERENCES}

[1] M. Berger, A. Tagliasacchi, and S. Lee, "State of the art in surface reconstruction from point clouds," Eurographics Star Reports, vol. 1, no. 1, 2014.

[2] R. Gomes, B. Silva, and L. Rocha, "Efficient 3d object recognition using foveated point clouds," Computers \& Graphics, vol. 37, no. 5, pp. 496-508, 2013.

[3] D. Aliaga, G. Daniel, and B. Bedrich, "Coupled segmentation and similarity detection for architectural models," Acm Transactions on Graphics, vol. 34, no. 4, p. 104, 2015.

[4] S. Ochmann, V. Richard, and K. Reinhard, "Automatic reconstruction of parametric building models from indoor point clouds," Computers \& Graphics, vol. 54, no. C, pp. 94-103, 2015.

[5] L. Guo, L. Liu, and H. Zheng, "Analysis, reconstruction and manipulation using arterial snakes," Acm Transactions on Graphics, vol. 29, no. 6, p. 152, 2010.

[6] H. Huang, S. Wu, and C. Daniel, "L1-medial skeleton of point cloud," Acm Transactions on Graphics, vol. 32, no. 4, pp. 1-8, 2013.

[7] A. Tagliasacchi, M. Olson, and H. Zhang, "Vase: Volume-aware surface evolution for surface reconstruction from incomplete point clouds," in Computer Graphics Forum, 2011, pp. 1563-1571.

[8] Y. Zhang, G. Geng, and X. Wei, "A statistical approach for extraction of feature lines from point clouds," Computers \& Graphics, vol. 56, no. C, pp. 31-45, 2016.

[9] Y. Lipman, C. Daniel, and L. David, "Parameterizationfree projection for geometry reconstruction," $\mathrm{Acm}$ Transactions on Graphics, vol. 26, no. 3, p. 22, 2007.

[10] H. Huang, S. Wu, and M. Gong, "Edge-aware point set resampling," Acm Transactions on Graphics, vol. 32, no. 1, p. 9, 2013.

[11] Y. Fan, M. Wang, N. Geng, D. He, J. Chang, and J. J. Zhang, "A self-adaptive segmentation method for a point cloud," The Visual Computer, pp. 1-15, 2017.

[12] K. Yin, H. Huang, and H. Zhang, "Morfit:interactive surface reconstruction from incomplete point clouds with curve-driven topology and geometry control," Acm Transactions on Graphics, vol. 33, no. 6, pp. 1-12, 2014. 